\title{
Regulation of UGT2A1 by miR-196a-5p and miR-196b-5p
}

\author{
Aimee K. Sutliff, Christy J. W. Watson, Gang Chen, and () Philip Lazarus \\ Department of Pharmaceutical Sciences, College of Pharmacy and Pharmaceutical Sciences, Washington State University, \\ Spokane, Washington
}

Received December 17, 2018; accepted February 15, 2019

\begin{abstract}
UDP-glucuronosyltransferase (UGT) 2A1 is an important enzyme in the detoxification of polycyclic aromatic hydrocarbons found in cigarette smoke. This enzyme is expressed in aerodigestive tract tissues including lung as both its wild-type and exon 4-deleted splice variant isoforms, with the latter acting as a negative regulator of wild-type UGT2A1 activity. UGT2A1 regulation may also be mediated by microRNA (miRNA). To identify miRNA important in the regulation of UGT2A1, expression analysis in tandem with in silico analysis suggested miR-196a$5 p$ and miR-196b-5p as potential top candidates. Significant reductions in firefly luciferase activity were observed in human embryonic kidney cell line 293 cells cotransfected with the wildtype UGT2A1 3' -untranslated region (UTR)-containing luciferase plasmid and either miR-196a-5p (62\%, $P=0.00080)$ or miR$196 \mathrm{~b}-5 \mathrm{p}(60 \%, P=0.00030)$ mimics. In pull-down assays, there was a 3.4- and 5.2-fold increase in miR-196a-5p $(P=0.054)$ and
\end{abstract}

miR-196b-5p $(P=0.035)$, respectively, using the UGT2A1 $3^{\prime}-$ UTR biotinylated mRNA probe as compared with the $\beta$-actin coding region control mRNA probe. UGT2A1 mRNA was reduced by $25 \%(P=0.058)$ and $35 \%(P=0.023)$ in $\mathrm{H} 146$ and $\mathrm{H} 1944$ cells, respectively, after overexpression of the miR196a-5p mimic. A similar 32\% $(P=0.030)$ and $41 \%(P=0.016)$ reduction was observed after over-expression of the miR-196b-5p mimic. In $\mathrm{H} 146$ cells transfected with miRNA mimic together with a small interfering RNA (siRNA) specific for the UGT2A1 splice variant, a significant reduction in 3-hydroxy-benzo[a]pyrene-glucuronide formation was observed. The miR-196a-5p- and miR-196b$55 p$-treated cells exhibited reductions of $35 \%(P=0.047)$ and $44 \%(P=0.0063)$, respectively. These data suggest that miR196a-5p and miR-196b-5p play an important role in UGT2A1 regulation within the lung and potentially other aerodigestive tract tissues.

\section{Introduction}

The UDP-glucuronosyltransferases (UGTs) are a family of phase II metabolizing enzymes that play an important role in the metabolism of endogenous compounds, including steroid hormones and bile acids as well as a significant number of drugs and environmental carcinogens (Tukey and Strassburg, 2000; Guillemette, 2003). UGTs conjugate the sugar cosubstrate, usually UDP-glucuronic acid, to a nonpolar substrate, creating a water-soluble metabolite that can be transported out of the cell and excreted from the body (Meech and Mackenzie, 1997). They are well-expressed in the liver but are also expressed in many other tissues including the brain, aerodigestive tract tissues, kidney, and reproductive organs (Court et al., 2012). Each of the 22 members of the UGT family of enzymes possesses both overlapping as well as unique

Funding for these studies were provided by the National Institutes of Health, National Institutes of Environmental Health Sciences [Grant R01ES025460] to P.L., and the Health Sciences and Services Authority of Spokane, WA [Grant WSU002292] to WSU College of Pharmacy and Pharmaceutical Sciences.

https://doi.org/10.1124/jpet.118.255935.

S This article has supplemental material available at jpet.aspetjournals.org. substrate specificities (Tukey and Strassburg, 2000). Therefore, certain UGTs may be considered critical for their role in detoxification. For instance, UGT1A1 is the only enzyme that removes bilirubin from the body, and failure to do so can cause jaundice in mild cases and severe developmental delays when UGT1A1 is nonfunctional or not expressed (Bosma et al., 1992).

Previous studies have shown that UGT2A1 is expressed in lung as well as other aerodigestive tract tissues and plays a role in the local detoxification of carcinogenic polycyclic aromatic hydrocarbons [PAHs; (Bushey et al., 2011)]. PAHs are the product of incomplete combustion of organic compounds. Important sources of exposure are through the smoking of tobacco products, the consumption of charbroiled foods, and certain workplace exposures including smelting and the laying of asphalt (Lijinsky and Shubik, 1964; Binet et al., 2002; Friesen et al., 2007). Glucuronidation by the UGT family of enzymes is an important detoxification mechanism for PAHs, and UGT2A1 appears to be the major UGT expressed in lung with activity against a broad range of simple and complex PAHs (Bushey et al., 2011).

Expression of UGT2A1 is subject to interindividual variability (Bushey and Lazarus, 2012). A well-expressed but

ABBREVIATIONS: 3-OH-BaP, 3-hydroxy-benzo[a]pyrene; Ct, cycle threshold; DMEM, Dulbecco's minimum essentials medium; HEK293, human embryonic kidney 293 cell line; HuH-7, human hepatocellular carcinoma cell line; miRNA, microRNA; MRE, miRNA recognition element; NCl-H146, human small cell carcinoma cell line; NCl-H1944, human non-small cell lung cancer cell line; PAHs, polycyclic aromatic hydrocarbons; PCR, polymerase chain reaction; qRT-PCR, quantitative reverse-transcription polymerase chain reaction; RISC, RNA-induced silencing complex of proteins; SDM, site-directed mutagenesis; siRNA, small interfering RNA; UDPGA, uridine diphosphoglucuronic acid; UGT, UDP-glucuronosyltransferase; UTR, untranslated region. 
functionally inactive splice variant was described for UGT2A1 and was shown to negatively regulate wild-type UGT2A1 activity by dimerization (Bushey and Lazarus, 2012). Such negative regulation of wild-type UGT2A1 expression could therefore potentially be associated with higher risk for $\mathrm{PAH}$ induced cancers in tissues with high levels of UGT2A1 splice variant expression because UGT2A1 was found to be the only UGT well-expressed in the lung with activity against the carcinogenic PAHs (Bushey and Lazarus, 2012).

MicroRNA (miRNA) are short nucleotide sequences that can reduce translation by binding to the $3^{\prime}$ untranslated region (UTR) of mature mRNA. They have critical roles in development because they tend to fine-tune expression, gradually reducing mRNA translation as specific stages of development come to an end (Lee et al., 1993). Additionally, their role in posttranscriptional regulation is complicated and has been explored in many diseases where overexpression or underexpression of miRNA leads to dysregulation of gene expression. For instance, lowered expression of a miRNA that suppresses the pleomorphic adenoma gene 1 oncogene enables overexpression and begins a cascade of events leading to tumor development (Pallasch et al., 2009).

Although no prior studies have investigated the possible regulation of UGT2A1 by miRNA, previous work has focused on the miRNA regulation of several members of the UGT1A and the UGT2B subfamilies. UGTs 1A1, 1A3, 1A6, and possibly $1 \mathrm{~A} 9$ were shown to be regulated by miR-491-3p (Dluzen et al., 2014), and UGTs 2B4, 2B7, and 2B10 expression were shown to be regulated by miR-216b-5p (Dluzen et al., 2016). Two separate studies identified miR-376c as a regulator of UGTs 2B15 and 2B17 (Wijayakumara et al., 2015; Margaillan et al., 2016). Additionally, two separate studies identified miR141-3p as a regulator of UGT1A (Papageorgiou and Court, 2017a; Tatsumi et al., 2018); however, one of the studies also identified miR-103b, miR-200a-3p, miR-376b-3p, and miR-1286 as capable of repressing UGT1A expression and miR-21-3p as a repressor of the variant UGT1A $3^{\prime}$-UTR (Papageorgiou and Court, 2017a). Thirteen miRNA were identified as potential repressors of UGT2B15 protein translation (Wijayakumara et al., 2015; Margaillan et al., 2016; Papageorgiou and Court, 2017b), seven were identified for UGT2B7 (Dluzen et al., 2016; Papageorgiou and Court, 2017b; Wijayakumara et al., 2017), and three were identified for UGT2B4 (Dluzen et al., 2016; Wijayakumara et al., 2017). The present study is the first to explore the potential regulation of UGT2A1 by miRNA.

\section{Materials and Methods}

Chemicals and Reagents. The pGL3-Promoter and pRL-TK renilla plasmids and the Dual-Reporter Assay System were obtained from Promega (Madison, WI). All synthesized DNA oligos used for 3'-UTR amplification, site-directed mutagenesis (SDM), and polymerase chain reaction (PCR) analysis were from Integrated DNA Technologies, Inc. (Coralville, IA). Lipofectamine 2000 and Lipofectamine 3000 transfection reagent were from Life Technologies (Carlsbad, CA). We purchased miRVana miRNA mimic miR-196a-5p (\#4464066, MC10068), miR-196b-5p (\#4464066, MC12946), and the negative control \#1 mimic (\#4464058) from Ambion (Austin, TX). The T7 MAXIscript Transcription Kit and NucAway Spin Columns were also Ambion products. TaqMan assays for UGT2A1 (Hs00792016_m1) and the endogenous control, ribosomal protein lateral stalk subunit P0 (RPLP0; 433376IF), were purchased from Applied Biosystems (Foster
City, CA), and the GeneJET Gel Extraction and DNA Cleanup Micro Kit were purchased from Thermo Scientific (Waltham, MA). The Ago2 antibody was purchased from MilliporeSigma (Burlington, MA). The following items were purchased from Invitrogen (Carlsbad, CA): Purelink RNA Mini Kit, SuperScript VILO cDNA Synthesis Kit, Biotin-14-CTP, and Dynabeads M-280 Streptavidin Beads. The iTaq Universal SYBR Green Supermix kit was purchased from Bio-Rad Laboratories (Hercules, CA), and PerfeCta SYBR Green Supermix and qScript microRNA cDNA Synthesis Kit were both purchased from Quanta BioSciences (Beverly, MA). The QuikChange II Site Directed Mutagenesis kit was obtained from Agilent Technologies (Santa Clara, CA), and uridine diphosphate glucuronic acid (UDPGA) and alamethicin were purchased from Sigma-Aldrich (St. Louis, MO). We purchased 3-hydroxy-benzo[a]pyrene (3-OH-BaP) from Toronto Research Chemical (Toronto, ON, Canada). All other chemicals used were obtained from Fisher Scientific (Pittsburg, PA) unless otherwise specified.

Cells and Culture Conditions. The human embryonic kidney cell line 293 (HEK293), the human non-small cell lung cancer cell line NCI-H1944, and the human small cell carcinoma cell line NCI-H146 were all purchased from the American Type Culture Collection (ATCC, Manassas, VA). The human hepatocellular carcinoma cell line HuH-7 was a kind gift from Dr. Jianming Hu (Penn State Hershey College of Medicine, Hershey, PA). HuH-7 and HEK293 cells were cultured in Dulbecco's minimum essentials medium (DMEM; Gibco, Carlsbad, CA) supplemented with 10\% FBS (Atlanta Biologicals, Lawrenceville, GA) and 1\% penicillin/streptomycin (Gibco). H1944 and H146 cells were cultured in RPMI 1640 (Gibco) supplemented with $10 \% \mathrm{FBS}, 1 \%$ penicillin/strep. The BEAS-2B human lung cell line was grown in plates treated with fibronectin and cultured using DMEM with $10 \%$ FBS but no penicillin/streptomycin. IMR-90 human lung cell line was grown in DMEM with $10 \%$ FBS and 1\% penicillin/ streptomycin while the A549 human lung cell line was grown in Ham's F-12K medium with $10 \% \mathrm{FBS}$ and $1 \%$ penicillin/streptomycin. All cells were grown and maintained at $37^{\circ} \mathrm{C}$ with $5 \% \mathrm{CO}_{2}$.

Tissues and miRNA Isolation. Lung tissues were obtained from the Banner Sun Health Research Institute (Sun City, AZ). Lung tissue samples were taken from deceased Caucasian individuals who perished from non-lung-related diseases. There were nine men and four women, ranging in age from 76 to 90 years old. The tissues were disrupted using the TissueLyzer II (Qiagen) for 2 minutes at $25 \mathrm{~Hz}$, and the total RNA was isolated using the PureLink RNA Mini Kit (Invitrogen). RNA concentration was measured using a Nanodrop 2000c (Thermo Scientific).

miRNA Binding Site Predictions. Binding site predictions for the 3'-UTR of UGT2A1 were obtained using TargetScan (www.targetscan.org/ vert_61/). TargetScan predictions are largely based on seed region and Total Context+ score (Lewis et al., 2005; Agarwal et al., 2015).

Real-Time Quantitative PCR. The synthesis of cDNA in the H146 and H1944 cell lines was performed using the SuperScript VILO cDNA Synthesis Kit and the Taqman gene expression assay was used to amplify UGT1A1 (Hs02511055_s1), UGT1A3 (Hs04194492_g1), UGT1A6 (Hs00153559_m1), UGT1A7 (Hs02517015_s1), UGT1A8 (Hs01592482_m1), UGT1A10 (Hs02516990_s1), UGT2A1 mRNA (Hs00792016_m1),UGT2A2 (Hs04195512_s1),UGT2B7 (Hs00426592_m1), UGT2B15 (Hs00870076_s1), UGT2B17 (Hs00854486_sH), and RPLP0 mRNA (Hs_99999902_m1). Per reaction, we used cDNA corresponding to $50 \mathrm{ng}$ of RNA. In 384-well plates using a CFX384 Thermocycler (BioRad Laboratories), we performed reactions for TaqMan assays in $15 \mu \mathrm{l}$ total volume as follows: one cycle of $95^{\circ} \mathrm{C}$ for 10 minutes, followed by 40 cycles of $95^{\circ} \mathrm{C}$ for 15 seconds, and $60^{\circ} \mathrm{C}$ for 1 minute. The cycle threshold $(\mathrm{Ct})$ values were determined using CFX Manager software (BioRad Laboratories). Gene expression was analyzed using the $2_{\mathrm{T}}^{-\Delta \Delta \mathrm{C}}$ method and compared with that observed for RPLP0 as an endogenous internal control (Livak and Schmittgen, 2001).

To amplify the UGT2A1 wild-type and splice variant cDNA sequences separate from one another in the H146 cell line, primers previously shown to amplify each isoform were used (see Supplemental 
Table 1; Bushey and Lazarus, 2012) along with an additional set of primers for the RPLP0 endogenous control (Supplemental Table 1). Each sequence was amplified individually in distinct wells to accurately quantify the expression levels of the wild-type and the splice variant. Amplified fragment sizes were kept between 228 and 237 nucleotides in length, and iTaq Universal SYBR Green Supermix kit was used to amplify the sequences. Ten-microliter reactions were amplified as follows: $95^{\circ} \mathrm{C}$ for 30 seconds followed 40 cycles of $95^{\circ} \mathrm{C}$ for 5 seconds, and $60^{\circ} \mathrm{C}$ for 15 seconds. To detect changes in the ratio of wild-type to splice variant mRNA levels, all gene expression data were normalized to the RPLP0 endogenous control.

To test for potential differences in wild-type versus splice variant UGT2A1 primer efficiencies, we isolated and purified genomic DNA from HEK293 cell lines overexpressing either splice variant or wildtype UGT2A1 (Bushey and Lazarus, 2012). Five concentrations of each cell line DNA were used for real-time quantitative PCR (qRTPCR) as the template for the corresponding primer pairs, starting at $40 \mathrm{ng}$ and decreasing 4 -fold for each dilution. We performed qRT-PCR as described in the Materials and Methods section for the H146 cell line, with the amplification cycling parameters repeated exactly as indicated. The log transformed DNA concentrations were plotted against $\mathrm{Ct}$ values to calculate the slope, with efficiency calculations based on the slope of the line.

We synthesized miRNA cDNAs using the qScript microRNA cDNA Synthesis Kit. PerfeCta SYBR Green SuperMix was used to amplify miR-196a-5p, miR-196b, miR-191-5p, and SNORD68 (see Supplemental Table 1 for primer sequences). The PerfeCta SYBR kit comes with a universal antisense primer that recognizes nucleotides added to the $3^{\prime}$ end during cDNA synthesis. The miRNA-specific sense primers used to amplify mature miRNA are provided in Supplemental Table 1. Quantitative PCR for miRNA was performed using cDNA corresponding to $37.5 \mathrm{ng}$ of total RNA in a CFX Thermocycler with the following conditions: $95^{\circ} \mathrm{C}$ for 2 minutes, followed by 40 cycles of $95^{\circ} \mathrm{C}$ for 10 seconds, and $60^{\circ} \mathrm{C}$ for 30 seconds. Each plate was run with no DNA template as a negative control.

For the miRNA expression analysis in human lung tissue specimens, expression was normalized to miR-191-5p, which has been previously demonstrated to exhibit the lowest expression variation of 15 miRNA assayed in a panel of 28 lung tumor and normal adjacent pairs, using the geNorm and NormFinder algorithms (Peltier and Latham, 2008). For miRNA expression analysis in the H146 and H1944 lung cancer cell lines, miR-196a-5p and miR-196b-5p expression was normalized to SNORD68, a small nucleolar RNA (SNORD) that has been previously shown to provide reliable normalization for miRNA quantification across cell lines (Das et al., 2016).

Construction of Reporter Plasmids. The luciferase reporter plasmids used in this study were constructed by inserting the UGT2A1 3'-UTR into the XbaI restriction cite located downstream of the luciferase reporter gene in the pGL3-Promoter vector. Briefly, using genomic DNA isolated from the Huh-7 cell line, we used primers modified with XbaI digestion sites at both ends (Supplemental Table 1) to amplify 603 nucleotides of the UGT2A1 3'-UTR corresponding to nucleotides +1736 to +2338 of the human UGT2A1 mRNA (NCBI Reference Sequence NM_006798.4). These nucleotides lie immediately adjacent to the UGT2A1 translation stop codon. The PCR-amplified region was cloned into the $\mathrm{XbaI}$ restriction site of the pGL3-promoter vector using standard protocols. The pGL3-UGT2A1 3'-UTR miRNA recognition element (MRE) seed deletion plasmid, which contained a deletion of six nucleotides within the seed sequence of the predicted miRNA binding region, was created by performing SDM using the QuikChange II Site Directed Mutagenesis Kit and sense and antisense primers described in Supplemental Table 1 using pGL3UGT2A1 3'-UTR as template. Confirmation of plasmid sequences was performed by Genewiz, LLC (South Plainfield, NJ).

Luciferase Assays. Twenty-four hours before transfection, HEK293 cells were seeded onto 24 -well plates at a density of approximately $5 \times 10^{4}$ cells/well. The pGL3 plasmids containing either the wild-type or miRNA seed deletion UGT2A1 3'-UTR sequences
(380 ng of each) were individually cotransfected with pRL-TK plasmid (20 ng, as a transfection efficiency control) along with $100 \mathrm{nM}$ of miR196a-5p mimic, miR-196b-5p mimic, or scrambled miRNA control using Lipofectamine 2000 transfection reagent. The HEK293 cells were harvested 24 hours after transfection using passive lysis buffer provided by the DLR kit. We measured the bioluminescent luciferase activity with a Bio-tek Synergy HT luminometer (Winooski, VT) using the Dual-Luciferase Reporter Assay System kit.

Biotin Pulldown Assays. The entire 3'-UTR of UGT2A1 was amplified by nested PCR using genomic DNA from HuH-7 cells. The initial PCR included sense and antisense primers located 235 nucleotides upstream of the UGT2A1 stop codon and 119 nucleotides downstream of the final UGT2A1 3'-UTR nucleotide, respectively. The 1312 nucleotide PCR fragment was gel-purified and subsequently used as template for nested PCR with primers designed to specifically amplify the entire UGT2A1 3 '-UTR. The sense primer corresponded to a location seven nucleotides upstream from the UGT2A1 stop codon and included the T7 promoter sequence at its $5^{\prime}$-end to allow for RNA synthesis of the corresponding UGT2A13'-UTR. The antisense primer corresponded to 17 nucleotides downstream of the final nucleotide within the UGT2A1 3'-UTR (see Supplemental Table 1).

The UGT2B10 3'-UTR was amplified from a pGL3 vector containing the UGT2B10 3 '-UTR described in a previous study (Dluzen et al., 2016), with the sense primer containing the T7 promoter sequence and the antisense primer beginning 1054 nucleotides downstream of the UGT2B10 stop codon (Supplemental Table 1). The $\beta$-actin coding region provided by the T7 MAXIscript kit was used as a negative control sequence.

UGT2A1 and UGT2B10 3'-UTR cDNA were isolated and purified using the GeneJET Gel Extraction and DNA Cleanup Kit. The T7 promoter-containing cDNAs provided the template to create biotinlabeled RNA using the T7 MAXIscript IVT Kit, performed following the manufacturer's protocols, with biotin-conjugated CTP added in a 1:4 ratio with unconjugated CTP. Reactions were subjected to DNase I digestion and the biotin-labeled RNA was purified using NucAway Spin Columns.

HuH-7 cells were lysed with polysomal lysis buffer $(100 \mathrm{mM} \mathrm{KCl}$, $5 \mathrm{mM} \mathrm{MgCl}_{2}, 10 \mathrm{mM}$ HEPES, pH 7.0, 0.5\% NP-40, RNase OUT) (Fan et al., 2011) and incubated with biotin-labeled RNA at room temperature for 30 minutes. Isolation and purification of miRNA bound to the biotin-labeled 3'-UTR RNA transcripts was performed using streptavidincoated magnetic Dynabeads. Lysates were added to the prepared beads and washed 3 times with PBS with $0.05 \%$ Tween 20 .

To analyze the RNA species bound to the biotinylated RNA, a phenol-chloroform extraction of the streptavidin beads was performed. Isolated miRNA were reverse transcribed using the qScript cDNA Synthesis Kit. The miRNA quantification was performed as described earlier using PerfeCta SYBR Green Supermix with primers specific for miR-196a-5p and miR-196b-5p (see Supplemental Table 1).

The pull-down experiment was repeated to collect protein bound to the 3 '-UTR complex. After isolation and purification of biotinylated $3^{\prime}$-UTR as described earlier, the beads were suspended in Trisbuffered saline and heated at $70^{\circ} \mathrm{C}$ for 10 minutes. To confirm the presence of proteins known to associate with the RNA-induced silencing complex of proteins (RISC), the Argonaute 2 (Ago2; part of the miRNA-binding complex; Pratt and MacRae, 2009) antibody was used in a Western blot analysis. Proteins isolated from the pull-down assays as well as HuH-7 cell lysate were run on $10 \%$ acrylamide gels and transferred to polyvinylidene fluoride membranes.

Glucuronidation Assays. Glucuronidation assays using homogenate from the H146 lung cancer cell line with 3-hydroxy-benzo[a]pyrene (3-OH-BaP) as substrate were performed to quantify UGT2A1 glucuronidation formation capacity in the presence of candidate miRNAs. Cells were transfected with varying concentrations of scrambled control or the miR-196a-5p or miR-196b-5p mimics using Lipofectamine 3000 . In an attempt to modify the expression of the UGT2A1 exon 4 splice variant protein within the cell line, a second set of cells were cotransfected with $10 \mathrm{nM}$ siRNA targeting the exon 3-5 
junction of the UGT2A1 splice variant (Fig. 1A) together with $100 \mathrm{nM}$ of either scrambled control, or the miR-196a-5p or miR-196b-5p mimics. As a negative control, a third set of cells similarly transfected with scrambled control or miRNA mimics was cotransfected with a scrambled siRNA. Cell homogenates were collected after 48 hours and subjected to five freeze-thaw cycles in liquid nitrogen before being passed through an 18-gauge needle a minimum of 10 times. BCA (bicinchoninic acid) assays were performed to quantify protein concentration in all homogenates.

Glucuronidation assays were performed after an initial incubation of $50 \mu \mathrm{g}$ homogenate with alamethicin $(50 \mu \mathrm{g} / \mathrm{mg}$ protein, in $100 \%$ ethanol) for 15 minutes on ice; the ethanol had been evaporated by allowing the aliquoted alamethicin to sit open to the air in the reaction tube for up to 30 minutes on ice before the addition of homogenate. Glucuronidation reactions were then performed in a final reaction volume of $25 \mu \mathrm{l}$ at $37^{\circ} \mathrm{C}$ with $50 \mathrm{mM}$ Tris- $\mathrm{HCl}$ (pH 7.4), $10 \mathrm{mM} \mathrm{MgCl}_{2}$, $4 \mathrm{mM}$ UDPGA, and $400 \mu \mathrm{M} 3-\mathrm{OH}-\mathrm{BaP}$, which is approximately 2 times the $K_{\mathrm{M}}$ for UGT2A1 against this substrate (Bushey et al., 2011). Reactions were terminated by the addition of $25 \mu \mathrm{l}$ cold acetonitrile on ice, and the reaction mixtures were centrifuged for 10 minutes at $16,100 \mathrm{~g}$ before the collection of supernatant.

Glucuronide formation was analyzed using a Sciex QTRAP liquid chromatography with tandom mass spectrometry system (Framingham, MA) with a 1.7- $\mu$ Acquity UPLC BEH C18 analytical column $(2.1 \times 50 \mathrm{~mm}$; Waters, Milford, MA $)$ in series with a $0.2-\mu \mathrm{m}$ Waters assay frit filter $(2.1 \mathrm{~mm})$. A gradient system consisting of solution A (5 $\mathrm{mM}$ ammonium acetate) and solution B (acetonitrile with $0.1 \%$ formic acid) was maintained at $0.4 \mathrm{ml} / \mathrm{min}$ to separate free substrate and the conjugated glucuronide. The initial conditions were $85 \% \mathrm{~A}$, transitioning to $5 \% \mathrm{~A}$ over the first 3.5 minutes of the gradient, returning to $85 \% \mathrm{~A}$ over the next 2.5 minutes, followed by an additional 1 minute at $85 \% \mathrm{~A}$.

Quantification of the 3-OH-BaP substrate used single reaction monitoring at $267.21 \mathrm{~m} / \mathrm{z}$; quantification of 3-OH-BaP-glucuronide used multiple reaction monitoring (MRM) in negative ion mode at the transition $443.21 \rightarrow 267.21 \mathrm{~m} / z$. The retention time for $3-\mathrm{OH}-\mathrm{BaP}$ and the 3-OH-BaP-glucuronide was 5.12 and 4.79 minutes, respectively. Reactions with HEK293 cells overexpressing UGT2A1 with and without UDPGA served as controls to identify the glucuronide peak. Quantification of glucuronidation was relative based on the ratio of peak area of 3-OH-BaP-glucuronide to 3-OH-BaP. Activity assays were performed in triplicate for all experiments.

Statistical Analysis. Statistical analysis was performed using GraphPad 5 software (La Jolla, CA). Statistical analysis employing the Student's $t$ test was performed for the analysis of results from luciferase assays, qPCR data from pulldown assays, data measuring mRNA levels, and glucuronidation activity assays. In addition, analysis of total UGT2A1 mRNA levels with varying concentrations of miRNA employed a linear trend test.

\section{Results}

In Silico Analysis of miRNA Binding to the 3'-UTR of UGT2A1 mRNA. TargetScan Release 6.2 was used to predict potential miRNA interactions with the UGT2A1 3'-UTR. The TargetScan algorithm predicted miR-196a-5p and miR-196b$5 p$ as potential candidates for the down-regulation of UGT2A1 (NM_006798) based on Context++ score, both scoring in the top five miRNA candidates. The miRNA recognition element (MRE) of miR-196a-5p and miR-196b-5p is located 269 nucleotides downstream of the UGT2A1 translational stop codon. The two miRNA species are identical in sequence except for a single nucleotide difference-an A in miR-196a-5p and a C in miR-196b-5p, 12 nucleotides from the $5^{\prime}$ end of the sequence (underlined in Fig. 1B). Of the five candidates predicted by TargetScan, only miR-196a-5p and miR-196b-5p contained

A

UGT2A1 wild type
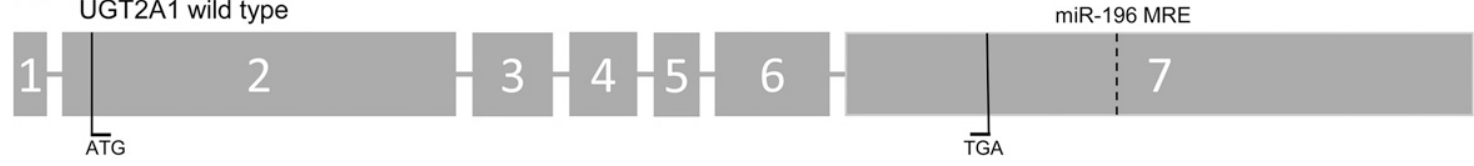

UGT2A1 splice variant

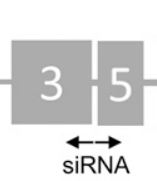

MiR-196 MRE
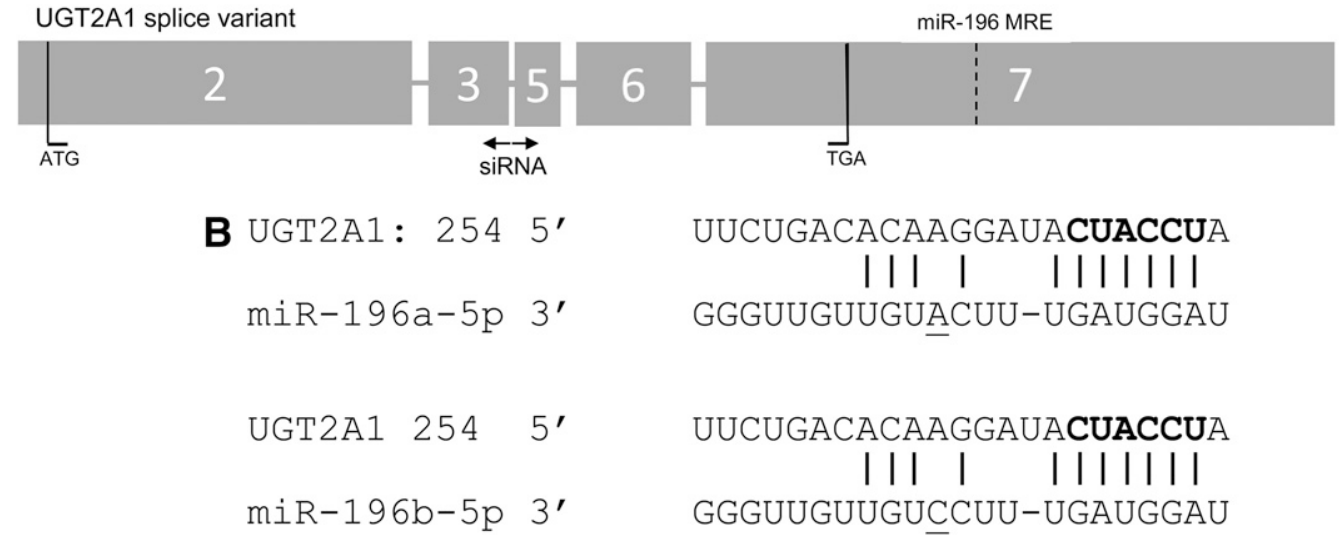

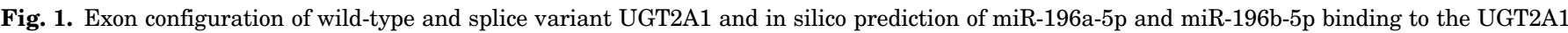

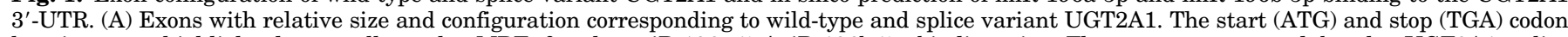

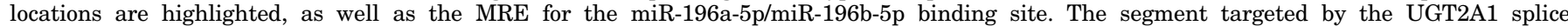

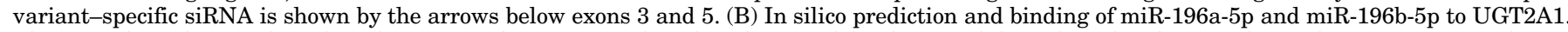

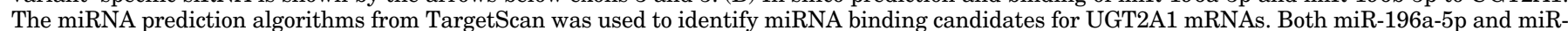

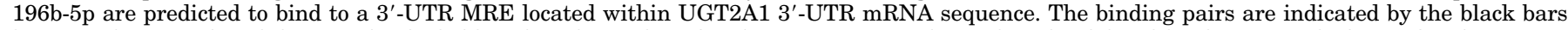

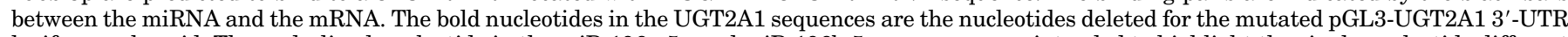

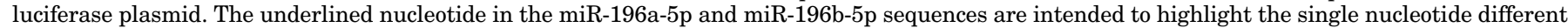
between the two mature miRNA sequences. 
additional predicted binding of nucleotides proximal to the MRE within the UGT2A1 mRNA (located at positions 13-16 of the miRNA), a feature suggested to potentially increase miRNA-mRNA targeting (Grimson et al., 2007).

In addition, multiple previous studies have shown miR196a-5p and miR-196b-5p expression in lung tissue (Hu et al., 2008; Tian et al., 2009; Hamamoto et al., 2013; Li et al., 2014), but no previous studies have supported a similar expression profile for the other miRNA listed as top-five candidates (miR-1231, miR-645, and miR-510-5p). Similarly, none of those three miRNA are expressed in lung adenocarcinoma samples (Panwar et al., 2017). Given that UGT2A1 is expressed in lung and may be an important enzyme involved in the local detoxification of tobacco carcinogens like the PAHs, the present study continued with a focus on miRNAs 196a-5p and 196b-5p.

In Vitro Binding Analysis of miR-196a-5p and miR196b-5p. Luciferase assays were performed with the firefly luciferase reporter gene to confirm the predicted interaction between the miRNAs and the 3'-UTR of UGT2A1. HEK293 cells were transfected with either pGL3-UGT2A1 3 '-UTR or the MRE seed deletion plasmid (six nucleotides deleted in the MRE; see the bold nucleotides in Fig. 1B). Along with the reporter plasmid, pRL-TK renilla was cotransfected as a control. There is no predicted binding site for the candidate miRNAs on this plasmid, and it exhibited little variation in its expression after transfection. In addition, each combination of reporter and control plasmid was cotransfected with $100 \mathrm{nM}$ of either miR-196a-5p mimic, miR-196b-5p mimic, or a scrambled control mimic.

There was a significant reduction in firefly luciferase activity in cells cotransfected with the wild-type UGT2A1 3'-UTR-containing plasmid in the presence of either miR-196a-5p (62\%, $P=0.00080)$ or miR-196b-5p (60\%, $P=0.00030)$ as compared with cells cotransfected with scrambled mimic (Fig. 2A). For both miR-196a-5p and miR-196b-5p, luciferase activity demonstrated significant or near-significant increases in activity for cells transfected with the UGT2A1 3'-UTR seed deletion mutant as compared with cells transfected with the wild-type UGT2A1 3 '-UTR sequence $(P=0.046$ and 0.075 for miR196a-5p and miR-196b-5p, respectively; Fig. 2A). There was a small but significant decrease in luciferase activity (29\%, $P=0.0030$ ) when cells were cotransfected with miR-196a-5p and the UGT2A1 3 '-UTR seed deletion mutant plasmid as compared with cells cotransfected with scrambled mimic control miRNA and the wild-type UGT2A1 3'-UTR sequence; no significant effect $(P=0.090)$ was observed for miR-196b-5p as compared with control (Fig. 2A).

When we compared cells transfected with the UGT2A1 3'UTR seed deletion mutant plasmid, a nonsignificant $20 \%$ decrease in luciferase activity was observed in cells cotransfected with miR-196a-5p as compared with cells cotransfected with scrambled mimic control miRNA. There was a nearidentical level of luciferase activity observed for cells cotransfected with miR-196b-5p as compared with scrambled mimic control miRNA in UGT2A1 3'-UTR seed deletion mutant plasmid-transfected cells (results not shown). These data suggest that both miRNA species bind to the predicted MRE of the UGT2A1 3'-UTR and are capable of altering UGT2A1 expression.

Binding of miR-196a-5p and miR-196b-5p to the $3^{\prime}$ UTR of UGT2A1. Quantitative PCR of RNA isolated from the pull-down assay measured levels of miR-196a-5p and miR196b-5p bound to either the mRNA probe for the UGT2A1 $3^{\prime}$-UTR versus a control mRNA probe corresponding to the $\beta$-actin coding region. The UGT2A1 3'-UTR probe was enriched 3.4- and 5.2-fold for miR-196a-5p $(P=0.054)$ and miR-196b-5p $(P=0.035)$, respectively, compared with the
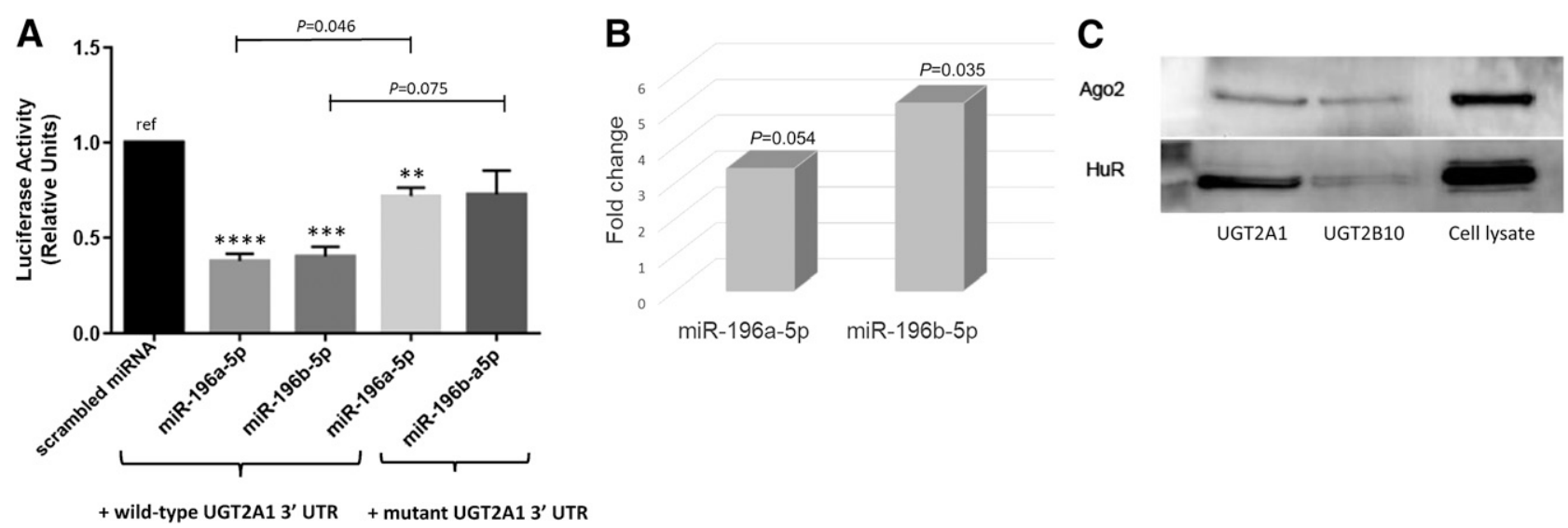

Fig. 2. UGT2A1 3'-UTR luciferase activity in the presence of miR-196a-5p and miR-196b-5p mimics and miR-196a-5p, miR-196b-5p association with the UGT2A1 3'-UTR. (A) The pGL3-UGT2A1 3'-UTR luciferase vector contains 603 nucleotides of the UGT2A1 3'-UTR, including the MRE for miR-196a-5p and miR-196b-5p. The pGL3-UGT2A1 3'-UTR MRE deletion vector contains a six nucleotide deletion within the miR-196a/b MRE seed sequence. UGT2A1 luciferase reporter vectors were cotransfected into HEK293 cells with the pRL-TK renilla control vector along with $100 \mathrm{nM}$ of miR-196a-5p, miR-196b-5p mimic, or scrambled miRNA control. Columns represent mean \pm S.E. of at least three independent experiments and are normalized to the scrambled miRNA-transfected control. The three columns on the left were performed using transfections of the luciferase vector containing the wild-type UGT2A1 3'-UTR; the two columns on the right were performed using the luciferase vector containing the UGT2A1 3'-UTR with the seed sequence deletion mutation. ${ }^{* *} P<0.05 ; * * * P<0.01 ; * * * * P<0.001$, all compared with the referent scrambled control miRNA cotransfected with the luciferase vector containing the wild-type UGT2A1 3'-UTR. (B) Biotinylated 3'-UTR probes of UGT2A1 or $\beta$-actin coding region were used to pull down endogenous miR-196a-5p or miR-196b-5p in HuH-7 cells. Each bar represents the amount of miR-196a-5p or miR-196b-5p bound to the UGT2A1 probe versus the $\beta$-actin probe measured by qRT-PCR after pull-down and isolation of the miRNA fraction from the two probes. Fold-change was based on $\mathrm{Ct}$ value of each miRNA measured from each probe. Columns represent the average fold change of three independent experiments. (C) A representative blot shows biotinylated 3'-UTR probes of UGT2A1 and UGT2B10 3'-UTRs that were used to pull down the protein, Ago2, to assess RISC binding. HuR binding was also assessed to confirm protein binding capacity of the 3'-UTR sequence. HuH-7 lysate served as a control for the two antibodies. 
$\beta$-actin coding region sequence, which is not predicted to bind to miR-196a-5p or miR-195b-5p (Fig. 2B). Further, Western blot analysis of in vitro pull-down experiments of miRNA and associated proteins demonstrated that Ago 2 was bound to the 3'-UTR of UGT2A1 as well as UGT2B10 (Fig. 2C), suggesting that miRNA-guided RISC is binding to the 3 '-UTR of both UGT mRNA species. Together, these data suggest that endogenous miR-196a-5p and miR-196b-5p are binding preferentially to the 3 '-UTR of UGT2A1 and provide further validation of the in silico prediction analysis.

Expression of UGT2A1 and miR-196a-5p or miR-196b$5 p$ in Lung Cell Lines and in Human Lung Tissue. Five human lung cell lines-H1944, H146, IMR-90, BEAS-2B, and A549-were screened for expression of UGTs that exhibited glucuronidating activity against $3^{\prime}-\mathrm{OH}-\mathrm{BaP}$ in previous studies (Dellinger et al., 2006; Bushey et al., 2011, 2013). Of the family 1A, 2A and 2B UGT enzymes, 1A4, 1A5, 2A3, 2B4, $2 \mathrm{~B} 10$ and 2B11 were shown to exhibit no detectable glucuronidation activity against $3^{\prime}-\mathrm{OH}-\mathrm{BaP}$ in previous studies and were not analyzed for expression in the current study. H146 and H1944 cells were the only two that exhibited detectable levels of UGT2A1 expression.

In H146 cells, UGT2A1 exhibited 3.2-fold higher levels of expression than the second-highest expressed UGT enzyme examined, UGT2A2 (Fig. 3A). The next highest levels of expression for UGT enzymes in H146 cells was for UGTs 2B15, 2B17, and 1A7, which were expressed at 77-, 98-, and 105-fold lower levels than UGT2A1. The opposite pattern was observed for H1944 cells, where UGT2A1 exhibited the lowest expression of all expressed UGTs (Fig. 3B). IMR-90, BEAS-2B, and A549 cells exhibited no detectable expression of UGT2A1 (data not shown). The levels of expression of UGT2A1 was $>10,000$-fold higher in H146 cells as compared with that observed in H1944 cells.

Both H146 and H1944 cells expressed miR-196a-5p and miR196b-5p, with H1944 cells exhibiting 19-fold higher levels of expression of miR-196a-5p and H146 exhibited 6.1-fold higher levels of miR-196b-5p (Fig. 3C). The total level of expression of both miRNA combined was similar between cell lines.

A panel of normal human lungs were examined for endogenous expression of miR-196a-5p and miR-196b-5p (Fig. 3D). In all samples, both miRNA were expressed at levels that were detectable by qPCR, with miR-196b-5p expressed at greater levels than miR-196a-5p in most samples. The difference between the lowest and highest expression of combined miRNA was 3-fold, while there was approximately a 4-fold change between the lowest and highest expression of each of the two individual candidate miRNAs.
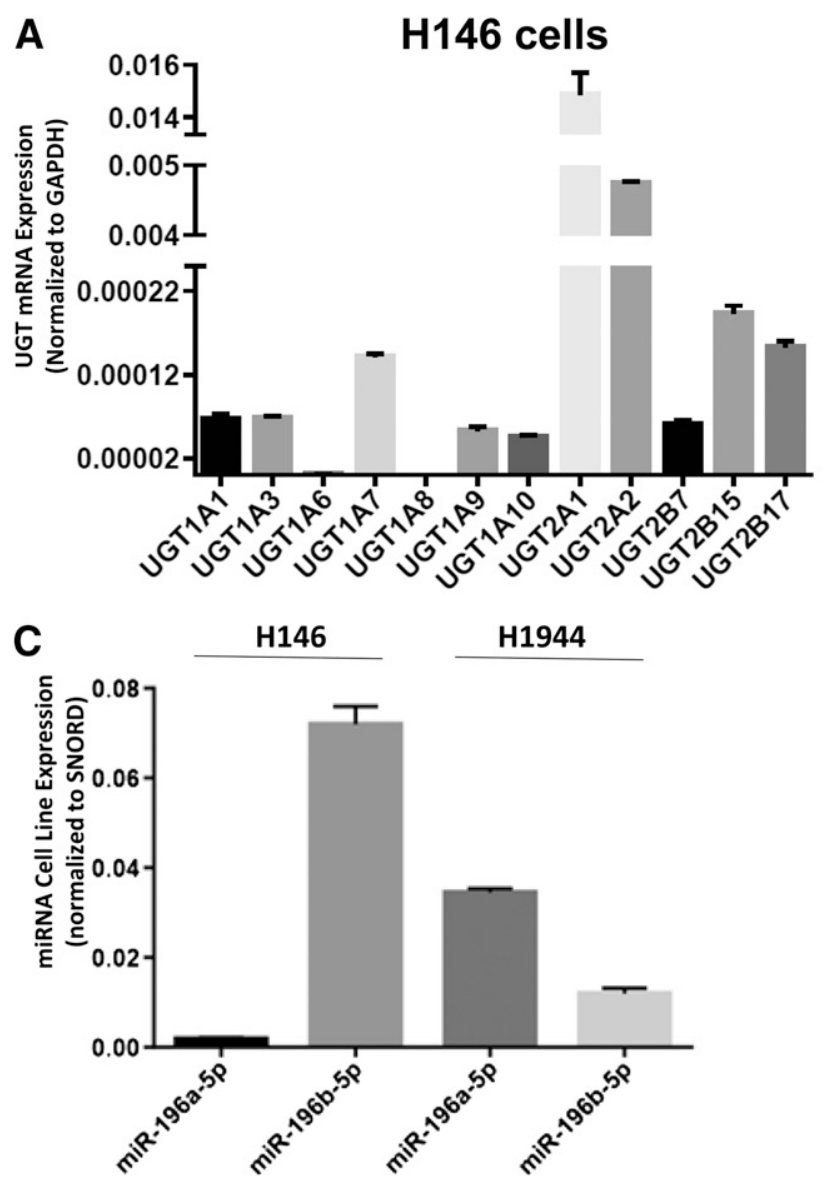

Fig. 3. Expression of UGT enzymes, miR-196a-5p, and miR-196b-5p. (A and B) UGT mRNA levels were quantified in H146 (A) and H1944 (B) cells by qRT-PCR. The mRNA expression was determined using GAPDH as an endogenous control. For H146 cells (A), the $y$-axis contains a gap between 0.00022 and 0.004 and 0.004 and 0.005 to better adjust for high UGT2A1 expression levels. For H1944 cells (B), the $y$-axis contains a gap between 0.0001 and 0.001 to better adjust for high UGT1A1 expression levels. (C) Expression levels of miR-196a-5p and miR-196b-5p were quantified by qRT-PCR in H146 and H1944 cells and were normalized to the levels of expression of the SNORD68 gene. (D) Expression levels of miR-196a-5p and miR-196b-5p in 13 individual human lung samples were quantified using qRT-PCR and were normalized to the levels of expression of miR-191-5p.
B H1944 cells

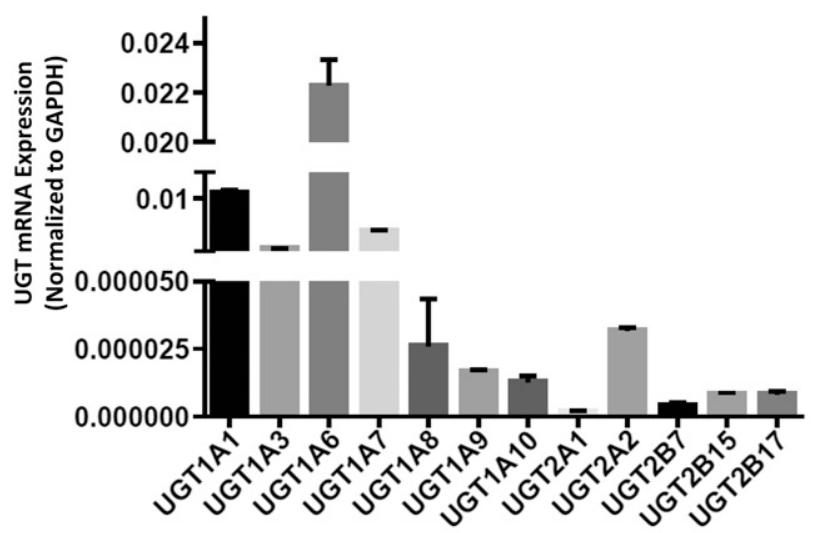

D

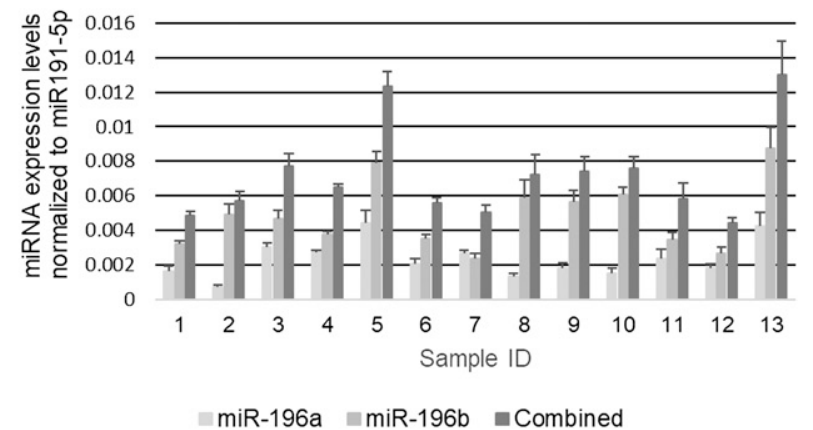


Effect of miRNA Mimics on UGT2A1 Expression in Cell Lines. We further investigated miR-196a-5p and/or miR-196b-5p interaction with the UGT2A1 3'-UTR by quantifying total UGT2A1 mRNA after transfecting miR-196a-5p or miR-196b-5p into H146 and H1944 cells (Fig. 4). Significant or near-significant decreases in UGT2A1 mRNA levels were observed in both cell lines after transfection with either miR196a-5p or miR-196b-5p. The largest decreases in UGT2A1 expression were observed at the highest concentration of miRNA mimic used in these experiments, with UGT2A1 mRNA reduced by $25 \%(P=0.058)$ and $35 \%(P=0.023)$ in H146 and H1944 cells, respectively, after overexpression of $100 \mathrm{nM}$ miR196a-5p mimic, and $32 \%(P=0.030)$ and $41 \%$ $(P=0.016)$ in $\mathrm{H} 146$ and $\mathrm{H} 1944$ cells, respectively, after overexpression of $100 \mathrm{nM}$ miR196b-5p mimic. Significant trends were observed toward decreasing UGT2A1 mRNA levels with increasing exogenous miRNA mimic for miR-196a-5p and miR-196b-5p in H146 $(P=0.022$ and $P=0.0091$, respectively $)$ and H1944 cells $(P=0.0065$ and $P=0.0011$, respectively). These data again suggest that both miR-196a-5p and miR-196b-5p play an important role in regulating UGT2A1 expression.

The UGT2A1 splice variant exhibits negative regulation on wild-type UGT2A1 glucuronidation activity. Previous studies have demonstrated that a 1:1 ratio of splice variant:wild-type expression will reduce wild-type UGT2A1 glucuronidation activity by 50\% (Bushey and Lazarus, 2012). Isoform specific primers (see Supplemental Table 1) were used to quantify the expression levels of wild-type and splice variant UGT2A1 isoforms in H146 cells. The primer efficiency for the wild type primers was $95.5 \%$; the efficiency for the splice variant primers was slightly lower, at $90.8 \%$.

As shown in Fig. 5, the ratio of splice variant to wild-type UGT2A1 mRNA in scrambled miRNA transfected H146 cells (2.4-fold; Fig. 5A) was slightly lower than H146 cells transfected with either miR-196a-5p or miR-196b-5p (2.9-fold for both miRNA). These high ratios of splice variant to wild-type UGT2A1 expression were not observed in tissues in previous studies (Bushey et al., 2013). The transfection of miR-196a-5p or miR-196b-5p was shown to significantly reduce the levels of wild-type UGT2A1 mRNA (miR-196a-5p: 49\%, $P=0.0074$; miR-196b-5p: $58 \%, P=0.0080$ ). A similar but smaller effect was observed for the UGT2A1 splice variant (miR-196a-5p: $37 \%, P=0.015$; miR-196b-5p: $43 \%, P=0.044$ ).

Effect of miR-196a-5p and miR-196b-5p on UGT2A1 Glucuronidation Activity in H146 Lung Cancer Cells. As described earlier, the H146 cell line expresses more than twice the amount of UGT2A1 splice variant mRNA as compared with wild-type UGT2A1 mRNA. In an attempt to investigate the role of miR-196a-5p and miR-196b-5p on wildtype versus splice variant UGT2A1 protein expression and activity, we designed a UGT2A1 splice variant-specific siRNA to decrease splice variant UGT2A1 mRNA levels relative to wild-type UGT2A1, with the siRNA crossing the boundary between exons 3 and 5 (Fig. 1A).

Wild-type UGT2A1 mRNA levels were not significantly reduced, but there was a $44 \%(P=0.0099)$ decrease in splice variant mRNA expression after transfection of $\mathrm{H} 146$ cells with $10 \mathrm{nM}$ UGT2A1 splice variant-specific siRNA as compared with cells transfected with $10 \mathrm{nM}$ scrambled siRNA (Fig. 5B). The UGT2A1 splice variant-specific siRNA reduced the ratio of splice variant to wild-type UGT2A1 mRNA from 2.6-fold higher than wild type to 1.6-fold higher than wild type.

Because 3-OH-BaP is an excellent substrate for UGT2A1 (Bushey et al., 2011) and because UGT2A1 is highly expressed in $\mathrm{H} 146$ cells relative to other UGTs (Fig. 3A), 3-OH-BaPglucuronide formation was used as a marker for UGT2A1 protein expression and activity in H146 cells. H146 cells were transfected with $100 \mathrm{nM}$ of scrambled miRNA control mimic, miR-196a-5p or miR-196b-5p. While no significant effect was observed for miR-196a-5p on 3-OH-BaP-glucuronide formation, a significant $(P=0.0013) 47 \%$ reduction in glucuronide formation was observed for miR-196b-5p (Fig. 5C).

In cells cotransfected with the UGT2A1 splice variant-specific siRNA plus scrambled control miRNA mimic, a near-significant $(P=0.052) 33 \%$ increase in 3-OH-BaP-glucuronide formation
A

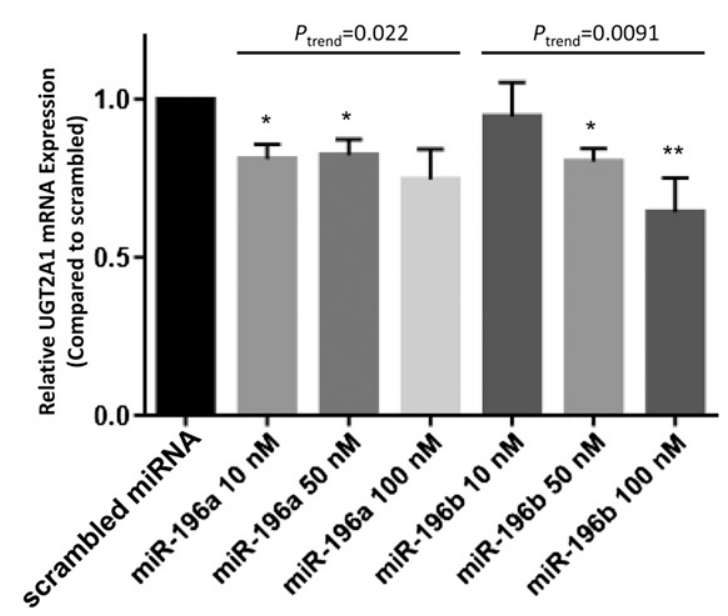

B

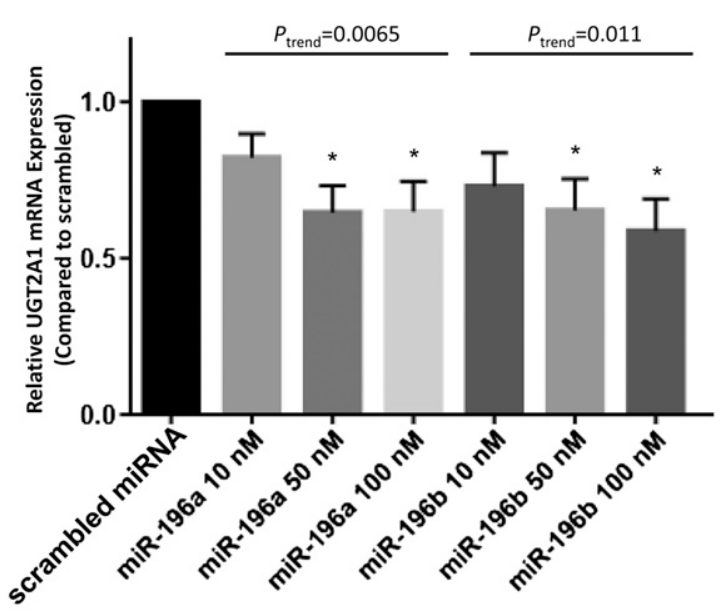

Fig. 4. UGT2A1 mRNA expression in H146 and H1944 cells after transfection with miR-196a-5p or miR-196b-5p mimics. UGT2A1 mRNA levels after transfection with 10, 50, or $100 \mathrm{nM}$ miR-196a-5p or miR-196b-5p were quantified by qRT-PCR relative to the endogenous internal control gene RPLP0 in H146 (A) and H1944 (B) cells. UGT2A1 mRNA levels are shown relative to the scrambled control miRNA mimic set at 1.0 as the reference. Columns represent mean \pm S.E. of three independent experiments. $* P<0.05 ; * * P<0.01$. 
A

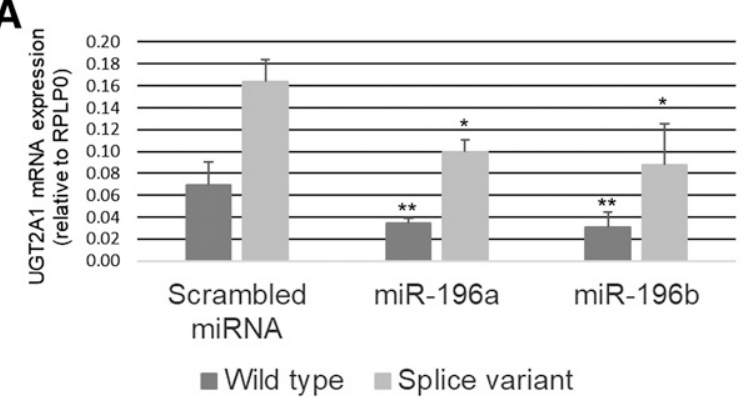

C

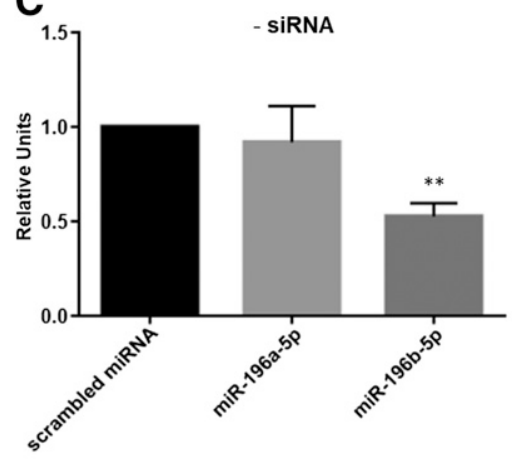

D
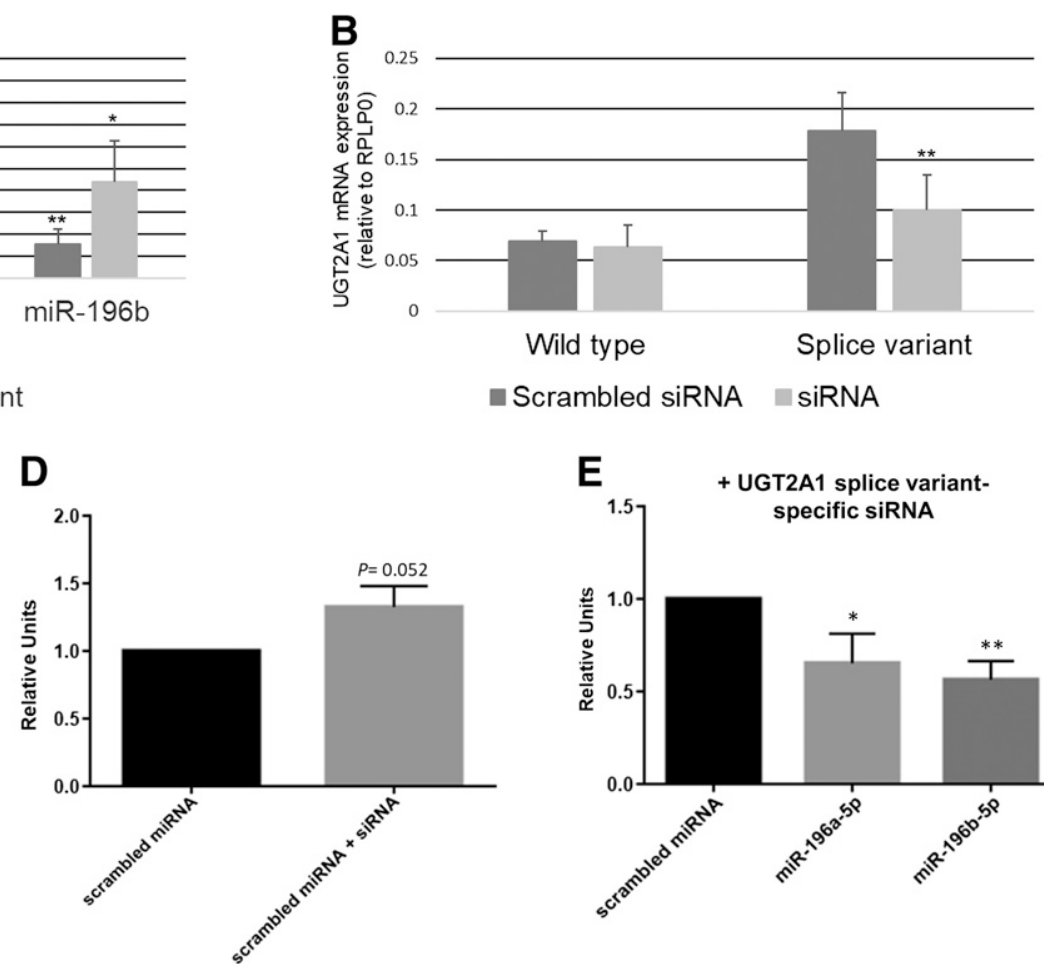

E

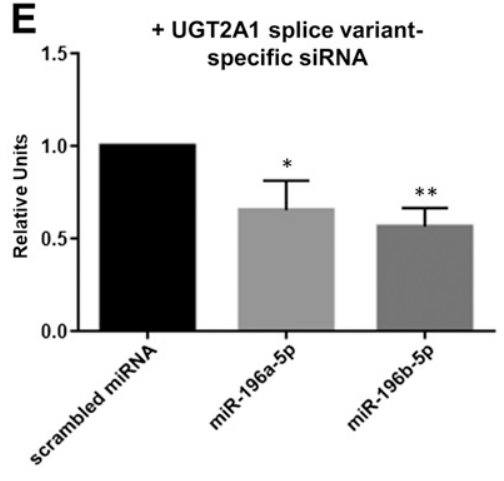

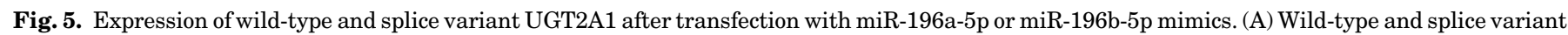

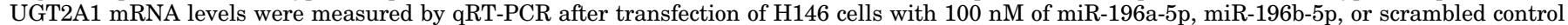

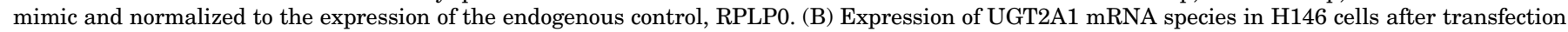

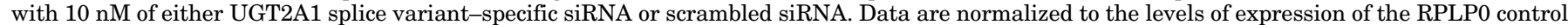

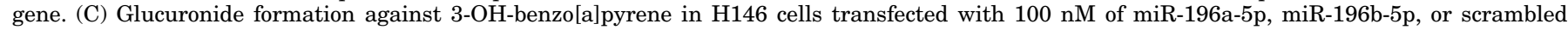

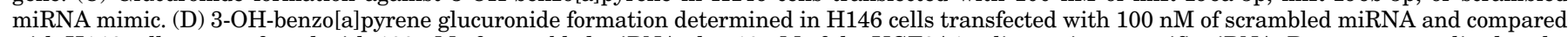

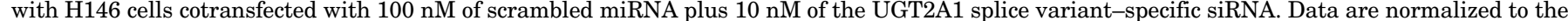

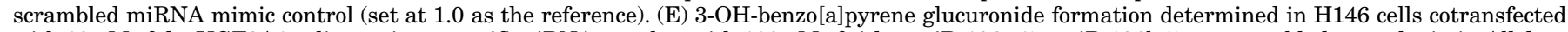

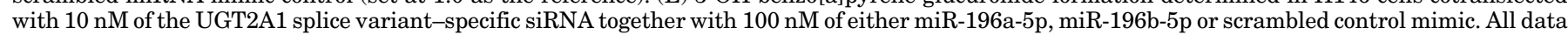

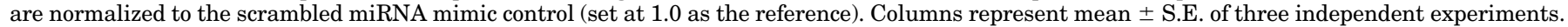
$* P<0.05 ; * * P<0.01$.

was observed as compared with cells transfected with the scrambled miRNA mimic alone (Fig. 5D). These data indicate some release of the negative regulation brought on by the interaction of the splice variant with the wild-type UGT2A1 enzyme.

H146 cells were then cotransfected with $100 \mathrm{nM}$ of either miR-196a-5p mimic, miR-196b-5p mimic, or scrambled miRNA control mimic together with the UGT2A1 splice variant-specific siRNA. In cells that were transfected with both the UGT2A1 splice variant-specific siRNA and miRNA mimic, a significant reduction in 3-OH-BaP glucuronide formation was observed in H146 cells cotransfected with either miR-196a-5p (35\%, $P=0.047)$ or miR-196b-55p (44\%, $P=0.0063$; Fig. 5E).

\section{Discussion}

This is the first study to explore miRNA regulation of UGT2A1, one of the only members of the UGT family that exhibits high activity against PAHs and is also expressed in a variety of aerodigestive tract tissues. It is the only UGT expressed in the lung that exhibits glucuronidation activity against a broad range of both simple and complex PAHs (Bushey et al., 2011).

In the present study, in silico analysis of the UGT2A1 3'UTR identified miR-196a-5p and miR-196b-5p as strong candidates for regulation of UGT2A1 by miRNA-based mechanisms.
The TargetScan program enables the identification of potential miRNA candidates based on an algorithm that considers 14 features of the target $3^{\prime}$-UTR, including supplementary pairing outside the seed region, local AU content, specific nucleotides in specific locations in the seed sequence, and site type (e.g., 8-mer vs. 7-mer; Agarwal et al., 2015). In a panel of human lung specimens collected from deceased individuals with no known lung pathologies, both miRNA species were expressed in all specimens, with miR-196b-5p exhibiting higher levels of expression than miR-196a-5p in 12 of the 13 specimens. Interestingly, the combination of the two miRNA exhibited a 3 -fold difference in total expression levels across the 13 specimens, suggesting that if they play a role in regulating UGT2A1 expression, there may be interindividual differences in this regulation. Analyzing a greater number of tissue samples may reveal the full extent of the expression differences of miR-196a-5p and miR-196b-5p in normal lung tissue.

Validation of the in silico predictions suggesting that miR-196a-5p and miR-196b-5p regulate UGT2A1 expression was observed in multiple experiments. When the $3^{\prime}$-UTR of UGT2A1 was inserted downstream of the firefly luciferase gene, both miR-196a-5p and miR-196b-5p demonstrated significant decreases in luciferase activity after cotransfection with the luciferase plasmid into HEK293 cells. The pull-down studies described in this study demonstrated specificity of binding of miR-196a-5p and miR-196b-5p to 
the UGT2A1 3'-UTR, with both miRNAs demonstrating 3 - to 5 -fold higher levels of binding to the mRNA corresponding to the UGT2A1 3'-UTR than a control mRNA species $(\beta$-actin). In addition, the significant decreases in endogenous UGT2A1 mRNA levels observed in H146 and H1944 cells after transfection with miR-196a-5p or miR-196b-5p further supported a potential regulatory effect of both miRNA on UGT2A1 expression.

Although lower levels of luciferase activity were observed after cotransfection of miR-196a-5p or miR-196b-5p with a luciferase plasmid containing a 6 -nucleotide deletion from the corresponding MRE of the UGT2A1 3'-UTR (as compared with scrambled mimic), the levels of activity were still significantly higher when compared with cotransfection with miR-196a-5p or miR-196b-5p mimics. The removal of the six nucleotides from the MRE changed the sequence from ACUACCUA to AAUUUUAA, making binding to this MRE less likely. However, it is possible that there was some promiscuous binding of miR-196a-5p and miR-196b-5p to the UGT2A1 3'-UTR sequence at a second location, leading to the slightly decreased levels of luciferase activity in the mutated MRE plasmids as compared with scrambled mimic controls.

A limitation of the present study is that direct protein quantification of UGT2A1 protein levels could not be performed due to a lack of sensitive antibodies. However, the data presented in this study are consistent with 3 '-OH-BaP glucuronidation capacity being a good marker of UGT2A1 activity in the $\mathrm{H} 146$ cell line; similar activity studies could not be performed in other cell lines including $\mathrm{H} 1944$ because of the low expression of UGT2A1 relative to other UGTs which also metabolize PAHs, including 3'-OH-BaP (Dellinger et al., 2006; Bushey et al., 2011, 2013). The only UGT that was shown in previous studies to exhibit 3-OH-BaP glucuronidating activity (Bushey et al., 2013) and approached a comparable level of expression to UGT2A1 in the H146 cell line in the present study was UGT2A2, which exhibited a 3.2-fold lower level of expression. The next most highest expressed UGTs in this cell line were UGTs $2 \mathrm{~B} 15,2 \mathrm{~B} 17$, and $1 \mathrm{~A} 7$, which exhibited between 77- and 105-fold lower levels of expression than UGT2A1.

These data suggest that UGT2A1 is the primary enzyme that is glucuronidating $3^{\prime}-\mathrm{OH}-\mathrm{BaP}$ in $\mathrm{H} 146$ cells and that UGT2A2 may also be playing a minor role. The significant decrease in 3-OH-BaP-glucuronide formation observed after transfection of miR-196a-5p or miR-196b-5p in H146 cells was consistent with the miR-196a-5p/miR-196b-5p-mediated decreases in UGT2A1 mRNA levels observed in both H146 and H1944 cells. These results further support a role for miR-196a$5 p$ and miR-196b-5p in directly regulating UGT2A1 expression and activity. It is possible that because they share the same 3 '-UTR, both UGTs $2 \mathrm{~A} 1$ and $2 \mathrm{~A} 2$ are regulated by miR-196a-5p and/or miR-196b-5p. This should be examined in future studies.

The splice variant of UGT2A1 was previously shown to be well-expressed in multiple aerodigestive tract tissues, has no endogenous glucuronidation activity, but was shown to oligomerize to the wild-type UGT2A1 isoform, resulting in decreased wild-type UGT2A1 glucuronidation activity (Bushey and Lazarus, 2012). The mRNA encoded by this variant (NM_001301239.1) is deleted in exons 1 and 4, with only exon 4 coding for amino acid sequence; exon 1 codes for entirely $5^{\prime}$-UTR sequences in the wild-type UGT2A1 mRNA (NM_006798.4). Unlike that observed previously in multiple human tissues (Bushey and Lazarus, 2012), the expression of the UGT2A1 splice variant was higher than that observed for wild-type UGT2A1 in the H146 cell line. In the present study, decreased expression of the splice variant and subsequent increases in $3^{\prime}-\mathrm{OH}-\mathrm{BaP}$-glucuronide formation were achieved using a custom-designed UGT2A1 splice variant-specific siRNA. In addition, decreased 3'-OH-BaPglucuronide formation was observed in $\mathrm{H} 146$ cells transfected with either miR-196a-5p or miR-196b-5p after cotransfection with the UGT2A1 splice variant-specific siRNA.

These results are consistent with the inhibition of wild-type and splice variant UGT2A1 mRNA expression observed with both miRNA. Predictions of the structure of the mRNA for both wild-type and splice variant UGT2A1 were performed by ViennaRNA (Supplementary Fig. 1; Lorenz et al., 2011). Although the overall structures differed for the two variants, their $3^{\prime}$-UTRs exhibited a highly similar structure prediction, a pattern consistent with the UGT2A1 inhibition observed with both miR-196a-5p and miR-196b-5p in this study. Significant decreases in $3^{\prime}-\mathrm{OH}-\mathrm{BaP}$-glucuronide formation were also observed for miR-196b-5p in cells not transfected with siRNA, but this inhibition pattern was not observed for miR-196a-5p in non-siRNA transfected cells, likely due to the high relative abundance of UGT2A1 splice variant mRNA in non-siRNA-transfected cells, rendering the experiments less sensitive.

The miR-196 family is a relatively well-studied family of miRNA (Chen et al., 2011). There are two identical miR-196a genes: miR-196a-1, located on chromosome 17, and miR196a-2, located on chromosome 12 . Only a single miR-196b gene exists, located on chromosome 7 . All three are located within HOX gene clusters and are conserved across many species (Fantini et al., 2015), and miR-196 play a critical role in embryonic development and in the regulation of HOX genes (Tanzer et al., 2005). The miR-196 family has been studied for regulation of a variety of other genes in multiple disease types. For example, miR-196b, miR-205, and miR375 were shown to serve as accurate biomarkers to distinguish squamous cell carcinoma from adenocarcinoma, with miR-196b found to be overexpressed in the squamous cell carcinoma samples (Hamamoto et al., 2013). Also, miR196a was shown to be a potential biomarker for head and neck, esophageal, pancreatic, non-small cell lung, and cervical cancers (Liu et al., 2012; Hou et al., 2014; Huang et al., 2014; Álvarez-Teijeiro et al., 2017; Fendereski et al., 2017), and both miR-196a and miR-196b are potential biomarkers for oral, gastric, and colorectal cancers as well as acute myeloid leukemia (Coskun et al., 2011; Lim et al., 2013; Liu et al., 2013; Mo et al., 2015). Because UGT2A1 is expressed in a variety of aerodigestive tract tissues including the lung, where both miR-196a-5p and miR196b-5p are also expressed, it is possible that these miRNAs are playing an important role in regulating its ability to detoxify PAHs from tobacco smoke and other exposures, thereby altering cancer risk in these tissues.

In summary, the data presented suggest a regulatory role for both miR-196a-5p and miR-196b-5p, acting on UGT2A1 in the lung. This regulation could impact the ability of UGT2A1 to detoxify carcinogens like PAHs and alter risk for developing lung cancer in response to chronic PAH exposure. 


\section{Acknowledgments}

We thank the Mass Spectrometry Core facility at Washington State University Spokane for their help with LC/MS.

\section{Authorship Contributions}

Participated in research design: Sutliff, Watson, Chen, Lazarus. Conducted experiments: Sutliff.

Contributed new reagents or analytic tools: Sutliff, Watson, Chen, Lazarus.

Performed data analysis: Sutliff, Watson, Chen, Lazarus.

Wrote or contributed to writing of the manuscript: Sutliff, Watson, Chen, Lazarus.

\section{References}

Agarwal V, Bell GW, Nam JW, and Bartel DP (2015) Predicting effective microRNA target sites in mammalian mRNAs. eLife 4:e05005.

Álvarez-Teijeiro S, Menéndez ST, Villaronga MA, Rodrigo JP, Manterola L, de Villalaín L, de Vicente JC, Alonso-Durán L, Fernández MP, Lawrie CH, et al. (2017) Dysregulation of Mir-196b in head and neck cancers leads to pleiotropic effects in the tumor cells and surrounding stromal fibroblasts. Sci Rep 7:17785.

Binet S, Pfohl-Leszkowicz A, Brandt H, Lafontaine M, and Castegnaro M (2002) Bitumen fumes: review of work on the potential risk to workers and the present knowledge on its origin. Sci Total Environ 300:37-49.

Bosma PJ, Chowdhury JR, Huang TJ, Lahiri P, Elferink RP, Van Es HH, Lederstein M, Whitington PF, Jansen PL, and Chowdhury NR (1992) Mechanisms of inherited deficiencies of multiple UDP-glucuronosyltransferase isoforms in two patients with Crigler-Najjar syndrome, type I. FASEB $J$ 6:2859-2863.

Bushey RT, Chen G, Blevins-Primeau AS, Krzeminski J, Amin S, and Lazarus P (2011) Characterization of UDP-glucuronosyltransferase 2A1 (UGT2A1) variants and their potential role in tobacco carcinogenesis. Pharmacogenet Genomics 21: 55-65.

Bushey RT, Dluzen DF, and Lazarus P (2013) Importance of UDP-glucuronosyltransferases 2A2 and 2A3 in tobacco carcinogen metabolism. Drug Metab Dispos 41:170-179.

Bushey RT and Lazarus P (2012) Identification and functional characterization of a novel UDP-glucuronosyltransferase 2A1 splice variant: potential importance in tobacco-related cancer susceptibility. J Pharmacol Exp Ther 343:712-724.

Chen C, Zhang Y, Zhang L, Weakley SM, and Yao Q (2011) MicroRNA-196: critical roles and clinical applications in development and cancer. J Cell Mol Med 15 $14-23$.

Coskun E, von der Heide EK, Schlee C, Kühnl A, Gökbuget N, Hoelzer D, Hofmann WK, Thiel E, and Baldus CD (2011) The role of microRNA-196a and microRNA$196 \mathrm{~b}$ as ERG regulators in acute myeloid leukemia and acute T-lymphoblastic leukemia. Leuk Res 35:208-213.

Court MH, Zhang X, Ding X, Yee KK, Hesse LM, and Finel M (2012) Quantitative distribution of mRNAs encoding the 19 human UDP-glucuronosyltransferase enzymes in 26 adult and 3 fetal tissues. Xenobiotica 42:266-277.

Das MK, Andreassen R, Haugen TB, and Furu K (2016) Identification of endogenous controls for use in miRNA quantification in human cancer cell lines. Cancer Genomics Proteomics 13:63-68.

Dellinger RW, Fang JL, Chen G, Weinberg R, and Lazarus P (2006) Importance of UDP-glucuronosyltransferase 1A10 (UGT1A10) in the detoxification of polycyclic aromatic hydrocarbons: decreased glucuronidative activity of the UGT1A10139Lys isoform. Drug Metab Dispos 34:943-949.

Dluzen DF, Sun D, Salzberg AC, Jones N, Bushey RT, Robertson GP, and Lazarus P (2014) Regulation of UDP-glucuronosyltransferase 1A1 expression and activity by microRNA 491-3p. J Pharmacol Exp Ther 348:465-477.

Dluzen DF, Sutliff AK, Chen G, Watson CJ, Ishmael FT, and Lazarus P (2016) Regulation of UGT2B expression and activity by miR-216b-5p in liver cancer cell lines. J Pharmacol Exp Ther 359:182-193.

Fan J, Ishmael FT, Fang X, Myers A, Cheadle C, Huang SK, Atasoy U, Gorospe M, and Stellato C (2011) Chemokine transcripts as targets of the RNA-binding protein $\mathrm{HuR}$ in human airway epithelium. J Immunol 186:2482-2494.

Fantini S, Salsi V, Vitobello A, Rijli FM, and Zappavigna V (2015) MicroRNA-196b is transcribed from an autonomous promoter and is directly regulated by $\mathrm{Cdx} 2$ and by posterior Hox proteins during embryogenesis. Biochim Biophys Acta 1849 1066-1080.

Fendereski M, Zia MF, Shafiee M, Safari F, Saneie MH, and Tavassoli M (2017) MicroRNA-196a as a potential diagnostic biomarker for esophageal squamous cell carcinoma. Cancer Invest 35:78-84.

Friesen MC, Demers PA, Spinelli JJ, Lorenzi MF, and Le ND (2007) Comparison of two indices of exposure to polycyclic aromatic hydrocarbons in a retrospective aluminium smelter cohort. Occup Environ Med 64:273-278.

Grimson A, Farh KK, Johnston WK, Garrett-Engele P, Lim LP, and Bartel DP (2007) MicroRNA targeting specificity in mammals: determinants beyond seed pairing. Mol Cell 27:91-105.

Guillemette C (2003) Pharmacogenomics of human UDP-glucuronosyltransferase enzymes. Pharmacogenomics J 3:136-158.

Hamamoto J, Soejima K, Yoda S, Naoki K, Nakayama S, Satomi R, Terai H, Ikemura $\mathrm{S}$, Sato T, Yasuda H, et al. (2013) Identification of microRNAs differentially expressed between lung squamous cell carcinoma and lung adenocarcinoma. $\mathrm{Mol}$ Med Rep 8:456-462.

Hou T, Ou J, Zhao X, Huang X, Huang Y, and Zhang Y (2014) MicroRNA-196a promotes cervical cancer proliferation through the regulation of FOXO1 and p27Kip1. Br J Cancer 110:1260-1268.

Hu Z, Chen J, Tian T, Zhou X, Gu H, Xu L, Zeng Y, Miao R, Jin G, Ma H, et al. (2008) Genetic variants of miRNA sequences and non-small cell lung cancer survival. $J$ Clin Invest 118:2600-2608.

Huang F, Tang J, Zhuang X, Zhuang Y, Cheng W, Chen W, Yao H, and Zhang S (2014) MiR-196a promotes pancreatic cancer progression by targeting nuclear factor kappa-B-inhibitor alpha. PLoS One 9:e87897.

Lee RC, Feinbaum RL, and Ambros V (1993) The C. elegans heterochronic gene lin-4 encodes small RNAs with antisense complementarity to lin-14. Cell 75:843-854.

Lewis BP, Burge CB, and Bartel DP (2005) Conserved seed pairing, often flanked by adenosines, indicates that thousands of human genes are microRNA targets. Cell 120:15-20.

Li X, Shi Y, Yin Z, Xue X, and Zhou B (2014) An eight-miRNA signature as a potential biomarker for predicting survival in lung adenocarcinoma. J Transl Med 12:159.

Lijinsky W and Shubik P (1964) Benzo(a)Pyrene and other polynuclear hydrocarbons in charcoal-broiled meat. Science 145:53-55.

Lim JY, Yoon SO, Seol SY, Hong SW, Kim JW, Choi SH, Lee JS, and Cho JY (2013) Overexpression of miR-196b and HOXA10 characterize a poor-prognosis gastric cancer subtype. World J Gastroenterol 19:7078-7088.

Liu CJ, Tsai MM, Tu HF, Lui MT, Cheng HW, and Lin SC (2013) miR-196a overexpression and miR-196a2 gene polymorphism are prognostic predictors of oral carcinomas. Ann Surg Oncol 20 (Suppl 3):S406-S414.

Liu XH, Lu KH, Wang KM, Sun M, Zhang EB, Yang JS, Yin DD, Liu ZL, Zhou J, Liu ZJ, et al. (2012) MicroRNA-196a promotes non-small cell lung cancer cell proliferation and invasion through targeting HOXA5. BMC Cancer 12:348.

Livak KJ and Schmittgen TD (2001) Analysis of relative gene expression data using real-time quantitative $\mathrm{PCR}$ and the $2(-$ delta delta $\mathrm{C}(\mathrm{T}))$ method. Methods 25: $402-408$.

Lorenz R, Bernhart SH, Höner Zu Siederdissen C, Tafer H, Flamm C, Stadler PF, and Hofacker IL (2011) ViennaRNA package 2.0. Algorithms Mol Biol 6:26.

Margaillan G, Lévesque É, and Guillemette C (2016) Epigenetic regulation of steroid inactivating UDP-glucuronosyltransferases by microRNAs in prostate cancer. J Steroid Biochem Mol Biol 155 (Pt A):85-93.

Meech R and Mackenzie PI (1997) Structure and function of uridine diphosphate glucuronosyltransferases. Clin Exp Pharmacol Physiol 24:907-915.

Mo JS, Alam KJ, Kang IH, Park WC, Seo GS, Choi SC, Kim HS, Moon HB, Yun KJ, and Chae SC (2015) MicroRNA 196B regulates FAS-mediated apoptosis in colorectal cancer cells. Oncotarget 6:2843-2855.

Pallasch CP, Patz M, Park YJ, Hagist S, Eggle D, Claus R, Debey-Pascher S, Schulz A, Frenzel LP, Claasen J, et al. (2009) miRNA deregulation by epigenetic silencing disrupts suppression of the oncogene PLAG1 in chronic lymphocytic leukemia. Blood 114:3255-3264.

Panwar B, Omenn GS, and Guan Y (2017) miRmine: a database of human miRNA expression profiles. Bioinformatics 33:1554-1560.

Papageorgiou I and Court MH (2017a) Identification and validation of microRNAs directly regulating the UDP-glucuronosyltransferase $1 \mathrm{~A}$ subfamily enzymes by a functional genomics approach. Biochem Pharmacol 137:93-106.

Papageorgiou I and Court MH (2017b) Identification and validation of the microRNA response elements in the 3 '-untranslated region of the UDP glucuronosyltransferase (UGT) $2 \mathrm{~B} 7$ and 2B15 genes by a functional genomics approach. Biochem Pharmacol 146:199-213.

Peltier HJ and Latham GJ (2008) Normalization of microRNA expression levels in quantitative RT-PCR assays: identification of suitable reference RNA targets in normal and cancerous human solid tissues. RNA 14:844-852.

Pratt AJ and MacRae IJ (2009) The RNA-induced silencing complex: a versatile genesilencing machine. J Biol Chem 284:17897-17901.

Tanzer A, Amemiya CT, Kim CB, and Stadler PF (2005) Evolution of microRNAs located within Hox gene clusters. J Exp Zoolog B Mol Dev Evol 304:75-85.

Tatsumi N, Tokumitsu S, Nakano M, Fukami T, and Nakajima M (2018) miR-141-3p commonly regulates human UGT1A isoforms via different mechanisms. Drug Metab Pharmacokinet 33:203-210.

Tian T, Shu Y, Chen J, Hu Z, Xu L, Jin G, Liang J, Liu P, Zhou X, Miao R, et al. (2009) A functional genetic variant in microRNA-196a2 is associated with increased susceptibility of lung cancer in Chinese. Cancer Epidemiol Biomarkers Prev 18: 1183-1187.

Tukey RH and Strassburg CP (2000) Human UDP-glucuronosyltransferases: metabolism, expression, and disease. Annu Rev Pharmacol Toxicol 40:581-616.

Wijayakumara DD, Hu DG, Meech R, McKinnon RA, and Mackenzie PI (2015) Regulation of human UGT2B15 and UGT2B17 by miR-376c in prostate cancer cell lines. J Pharmacol Exp Ther 354:417-425.

Wijayakumara DD, Mackenzie PI, McKinnon RA, Hu DG, and Meech R (2017) Regulation of UDP-glucuronosyltransferases UGT2B4 and UGT2B7 by MicroRNAs in liver cancer cells. J Pharmacol Exp Ther 361:386-397.

Address correspondence to: Dr. Philip Lazarus, Department of Pharmaceutical Sciences, College of Pharmacy and Pharmaceutical Sciences, Washington State University, PO Box 1495, Spokane, WA 99210. E-mail: phil.lazarus@wsu.edu 